\title{
Quantitative characterization of translational riboregulators using an in vitro transcription-translation system
}

\author{
Anis Senoussi, ${ }^{\dagger, \perp}$ Jonathan Lee Tin Wah, ${ }^{\dagger, \perp}$ Yoshihiro Shimizu, ${ }^{\ddagger}$ Jérôme

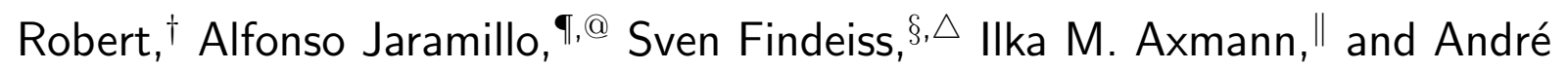 \\ Estevez-Torres ${ }^{*, \dagger}, \perp$ \\ †Sorbonne Université, Laboratoire Jean Perrin, F-75005, Paris, France \\ $\ddagger$ Laboratory for Cell-Free Protein Synthesis, RIKEN Quantitative Biology Center, 6-2-3, \\ Furuedai, Suita, Osaka 565-0874, Japan \\ \ Warwick Integrative Synthetic Biology Centre and School of Life Sciences, University of \\ Warwick, CV4 7AL, Coventry, UK \\ $\S$ Dept. Computer Science, and Interdisciplinary Center for Bioinformatics, University Leipzig, \\ Härtelstrasse 16-18, D-04107 Leipzig, Germany \\ \|Institute for Synthetic Microbiology, Cluster of Excellence on Plant Sciences (CEPLAS), \\ Heinrich Heine University Düsseldorf, Universitätsstrasse 1, 40225 Düsseldorf, Germany \\ $\perp U M R$ 8237, CNRS, F-75005, Paris, France \\ \#CNRS-UMR8030, Laboratoire iSSB and Université Paris-Saclay and Université d'Évry and \\ CEA, DRF, IG, Genoscope, Évry 91000, France \\ @ Institute for Integrative Systems Biology (I2SysBio), University of Valencia-CSIC, 46980

$$
\text { Paterna, Spain }
$$ \\ $\triangle$ University of Vienna, Faculty of Computer Science, Research Group Bioinformatics and \\ Computational Biology and Faculty of Chemistry, Department of Theoretical Chemistry, \\ Währingerstrasse 29, A-1090 Vienna, Austria
}

E-mail: andre.estevez-torres@sorbonne-universite.fr 


\section{${ }_{22}$ Keywords}

\begin{abstract}
Riboregulators are short RNA sequences that, upon binding to a ligand, change their secondary structure and influence the expression rate of a downstream gene. They constitute an attractive alternative to transcription factors for building synthetic gene regulatory networks because they can be engineered de novo. However, riboregulators are generally designed in silico and tested in vivo, which provides little quantitative information about their performances, thus hindering the improvement of design algorithms. Here we show that a cell-free transcription-translation (TX-TL) system provides valuable information about the performances of in silico designed riboregulators. We first propose a simple model that provides a quantitative definition of the dynamic range of a riboregulator. We further characterize two types of translational riboregulators composed of a cis-repressed (cr) and a trans-activating (ta) strand. At the DNA level we demonstrate that high concentrations of taDNA poisoned the activator until total shut off, in agreement with our model, and that relative dynamic ranges of riboregulators determined in vitro are in agreement with published in vivo data. At the RNA level, we show that this approach provides a fast and simple way to measure dissociation constants of functional riboregulators, in contrast to standard mobility-shift assays. Our method opens the route for using cell-free TX-TL systems for the quantitative characterization of functional riboregulators in order to improve their design in silico.
\end{abstract}

in vitro synthetic biology, RNA translational riboregulator, cell-free protein synthesis 
During the early wave of synthetic biology, ${ }^{1,2}$ known transcription factors were wired to their corresponding promoter sequences to control the expression of other transcription factors or effector proteins. While this approach has been very successful in engineering gene regulatory networks (GRNs) ${ }^{3}$ with few nodes, the number of different elements in the majority of synthetic GRNs has stagnated at $5-6,{ }^{4,5}$ although a remarkable example contains 11 elements. ${ }^{6}$ Two arguments may explain this limit. First, protein-DNA interactions are very difficult to design, although very promising computational methods are arising, ${ }^{7}$ and the engineer must thus choose well-known transcription factor-promoter pairs. Second, the expression of these transcription factors imposes a metabolic burden to the cells. ${ }^{8}$

Implementing regulatory circuits at the RNA level may help solving these issues essentially because RNA-RNA interactions can be predicted from the sequence. ${ }^{9-11}$ Moreover, in the case of transcriptional regulators, protein expression is not needed for regulation, which lowers the metabolic burden. ${ }^{12}$ The principal component of an RNA-regulated GRN is the riboregulator: an RNA sequence in the 5' untranslated region (UTR) of a gene of interest that has an effect on its expression rate. Since they were first used in synthetic biology more than a decade ago, ${ }^{13}$ several riboregulators have been designed and implemented in vivo, both in prokaryotic ${ }^{14-18}$ and eukaryotic cells. ${ }^{19}$ However, their design remains more difficult than expected and many implementations do not work in vivo. ${ }^{12}$ One reason to this is that structure-prediction tools do not yet precisely capture the complexity involved in the folding of RNA species several hundreds of nucleotides long. Furthermore, in silico design relies on a structural model of riboregulation, which needs to be transformed into predictable features in order to generate optimized sequences. Another reason is that it is hard to control and tune the copy number of plasmids or genes in vivo and thus testing new parts in vivo ${ }^{20,21}$ often provides information that is difficult to correlate with thermodynamic parameters used in silico.

Including a phase of in vitro testing in the workflow of engineering riboregulators could potentially solve these problems. Structural characterization of riboregulators ${ }^{22,23}$ helps as- 
sessing the correctness of the designed structures and has been recently combined with functional information ${ }^{24}$ but does not provide quantitative kinetic and thermodynamic data. To overcome these difficulties and accelerate the improvement of in silico designs, cell-free transcription-translation (TX-TL) platforms are an attractive tool for testing genetic regulatory modules in synthetic biology. ${ }^{5,25-27}$ Currently, there are two types of TX-TL systems available, cell-extract-based and PURE (Protein synthesis Using Recombinant Elements) systems. The first one is obtained by recovering the protein fraction from E. coli ${ }^{26}$ while the PURE is just composed of individually-purified recombinant elements necessary for expression in vitro. ${ }^{28,29}$ TX-TL in vitro testing can be used to qualitatively evaluate the performances of new designs in a faster manner ${ }^{5,27,30}$ or to provide quantitative data such as thermodynamic and kinetic rates ${ }^{31}$ that are of great value to improve in silico methods.

Here we used a PURE TX-TL platform to illustrate the second approach. Briefly, the PURE system includes T7 RNA polymerase (RNAP), an energy-coupling module for NTP regeneration, transfer RNAs, ribosomes and translation initiation, elongation and release factors in a suitable buffer. ${ }^{29,32}$ Its composition is well-controlled and it contains low levels of ribonucleases. The PURE system has already been used to characterize transcriptiontranslation dynamics ${ }^{33,34}$ and $\mathrm{GRNs}^{35}$ but has not yet been used to characterize riboregulators. Cell-extract TX-TL systems have been used to study transcriptional ${ }^{30,31}$ but not translational riboregulators. To the best of our knowledge, TX-TL systems have so far investigated GRNs that mix both transcriptional and translational dynamics. In this work, we characterize the dynamics of translational riboregulators at the DNA and RNA level, which allows to independently study transcription and translation and clearly pinpoint design shortcomings. The simplicity of the PURE system allows us to propose an analytical model of riboregulation that fits our data. The proposed model, together with the in vitro experiments, show that: i) TX-TL linearly amplifies the concentration of active RNA and quadratically amplifies the concentration of coding DNA, ii) we can provide a quantitative definition of the dynamic range of a riboregulator, $\rho_{O N / O F F}$, iii) $\rho_{O N / O F F}$ is a bell-shaped 
function of the concentration of regulatory DNA, iv) the relative values of $\rho_{O N / O F F}$ measured in vitro coincide with published data in vivo for four riboregulators out of five and v) we can reliably use TX-TL to measure the dissociation constant, $K_{d}$, between the two RNA species. Although these results were facilitated by the simplicity of the PURE system, they could be extended to other cell-free TX-TL systems and possibly in vivo. These quantitative insights on translational riboregulators may also help improving in silico design routines.

\section{Results and discussion}

\section{Translation rate vs. structure as the optimization goal for a ri- boregulator}

Our study focuses on translational riboregulators, which are composed of two RNA strands (Figure 1A). One of them, called cis repressed RNA, noted $\mathrm{R}_{c r}$, about 800 nucleotides (nt) long, codes for a gene but bears a hairpin in its 5'-untranslated region (5'-UTR) that prevents the ribosome to start translating the downstream gene. The other one, a small transactivating RNA, about $100 \mathrm{nt}$ long, noted $\mathrm{R}_{t a}$, hybridizes to the 5'-UTR of $\mathrm{R}_{c r}$, opens up the hairpin and forms an active complex, $\mathrm{R}_{a c t}$, increasing the translation rate.

Ultimately, the riboregulator engineer is interested in controlling the rate of translation for $\mathrm{R}_{c r}$ and $\mathrm{R}_{a c t}$, noted respectively $r_{t l}^{c r}$ and $r_{t l}^{a c t}$, and seek the objective $r_{t l}^{a c t} \gg r_{t l}^{c r} \approx 0$ for an activator (Figure 1B). For convenience we assign a species name to an RNA sequence, but one must bear in mind that a given RNA sequence, for instance $\mathrm{R}_{c r}$, may fold in an ensemble of different structures $\left\{\mathrm{R}_{c r}^{i}\right\}$, with different translation rates $\left\{r_{t l}^{c r, i}\right\}$. Current in silico design methods ${ }^{11,36}$ compute the ensemble of secondary structures $\left\{\mathrm{R}_{c r}^{i}, \mathrm{R}_{t a}^{j}, \mathrm{R}_{a c t}^{k}\right\}$ that minimizes free energy. However, the structure-to-function relationship that associates an RNA conformation with its translation rate is hard to establish. Thus, a set of heuristic rules attributes low values of translation rates $r_{t l}^{c r, j}$ to structures where the RBS or the start codon are buried in a hairpin (Figure 1), and high values of $r_{t l}^{a c t, k}$, to structures where 
(A)

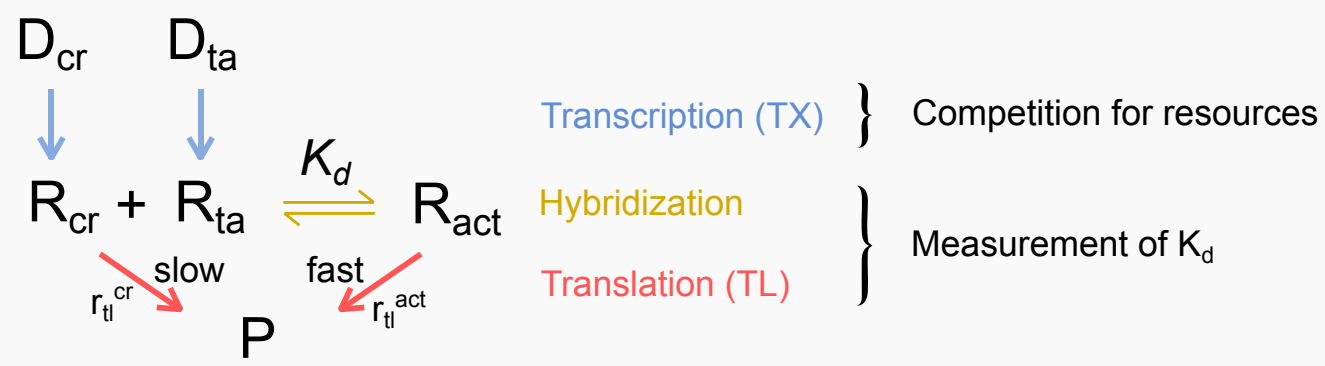

(B)

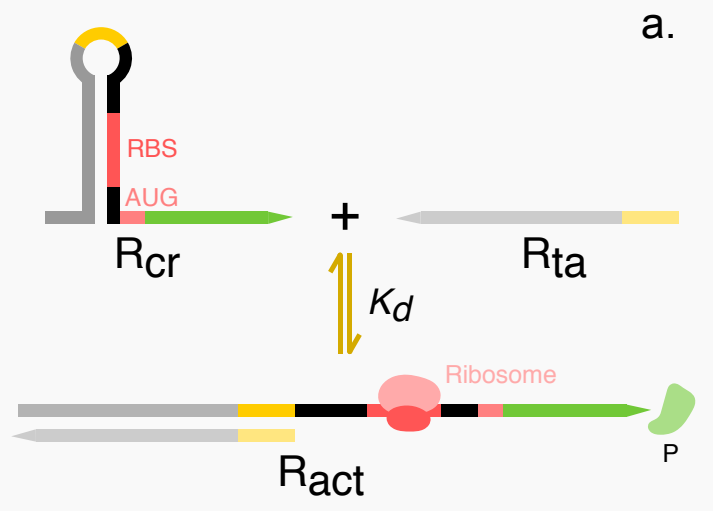

a.

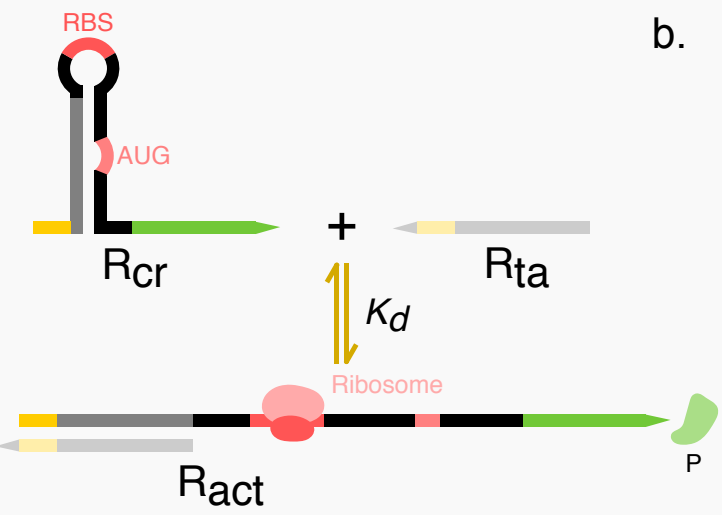

Figure 1: Principle of a translational riboregulator and of its characterization using a cell-free transcription-translation system (TX-TL). (A) Mechanism of transcription, riboregulation through RNA hybridization and translation used in this work. DNA sequences $\mathrm{D}_{c r}$ and $\mathrm{D}_{t a}$ are transcribed into a cis-repressed, $\mathrm{R}_{c r}$, and a trans-activator, $\mathrm{R}_{t a}$, RNA strands. $\mathrm{R}_{c r}$ may be slowly translated into protein $\mathrm{P}$ or hybridize with $\mathrm{R}_{t a}$ to form $\mathrm{R}_{a c t}$ that is translated more rapidly into $\mathrm{P}$. Measuring the dynamics of fluorescence production by a fluorescent protein $\mathrm{P}$ provides information about resource competition when evaluating the system at the DNA level and quantitative values of dissociation constants $K_{d}$ when RNA concentration is fixed. (B) Sketches of the two operation modes of translational riboregulators functioning as an activator. The 5'-UTR of $\mathrm{R}_{c r}$ RNA, forms a hairpin that hides either the ribosome binding site (RBS, a.) or the start codon (AUG, b.) away from the ribosome. $\mathrm{R}_{t a}$ hybridizes with $\mathrm{R}_{c r}$, unwinding the hairpin and liberating the RBS and/or the AUG promoting translation. 
these are accessible. However, these heuristic rules often fail. Moreover, minimizing the free energy of the RNA structures implies that the hypothesis of thermodynamic equilibrium holds, which is far from being true in vivo in the presence of co-transcriptional folding and RNA chaperones. ${ }^{37,38}$ To shed light into this problem we measured translation and expression (transcription and translation) dynamics of recently in silico designed riboregulators ${ }^{20,21}$ in the PURE system. ${ }^{28}$

\section{The TX-TL system linearly amplifies the concentration of RNA and quadratically amplifies the concentration of DNA}

We first characterized the translation and expression reactions of the PURE system in the absence of riboregulation. To do so, we prepared by PCR a linear DNA fragment coding for a green fluorescent protein (GFP) with no upstream regulatory region, called $\mathrm{cr}^{-} \mathrm{DNA}$. It is composed of a T7 RNAP promoter, a ribosome binding site, the GFP-coding sequence, and a $\mathrm{T} 7$ terminator. In addition, we prepared by in vitro transcription the corresponding messenger RNA, $\mathrm{cr}^{-} \mathrm{RNA}$, from $\mathrm{cr}^{-}$DNA. We successively used $\mathrm{cr}^{-} \mathrm{RNA}$ and $\mathrm{cr}^{-} \mathrm{DNA}$ as the coding nucleic acid input of the TX-TL system. We varied the concentration of the input and we measured the fluorescence emitted by the GFP produced over time (Figure 2). Starting from $\mathrm{cr}^{-} \mathrm{RNA}$, the translation module of the TX-TL system actively produced GFP during 2 hours. The translation kinetics displayed three different phases: during about 5 min no signal was discernable from the background level, then followed a phase of quasi-linear increase during $100 \mathrm{~min}$, that slowed down until a plateau was reached (Figure 2A). In the range $0-80 \mathrm{nM}$ of $\mathrm{cr}^{-} \mathrm{RNA}$, both the final intensity and the maximum rate of fluorescence growth, $v_{t l}^{\max }$, increased linearly with the initial quantity of coding RNA (Figure 2B). For higher concentrations there was a saturation: putting more RNA template did not increase significantly the final yield or the maximal production rate. When using $\mathrm{cr}^{-} \mathrm{DNA}$ as the initial input, the dynamics of the fluorescence intensity showed both common and contrasting features with the previous case (Figure 2C). Three phases were still observed: delay, growth 
(A)

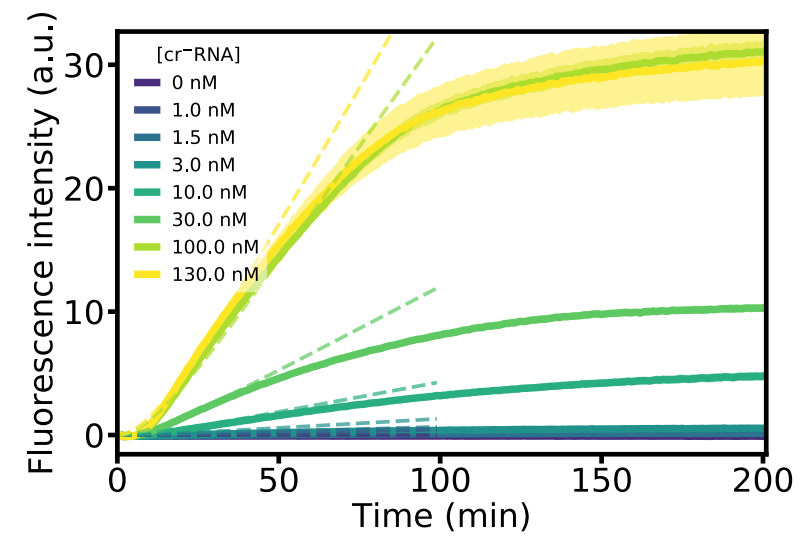

(C)

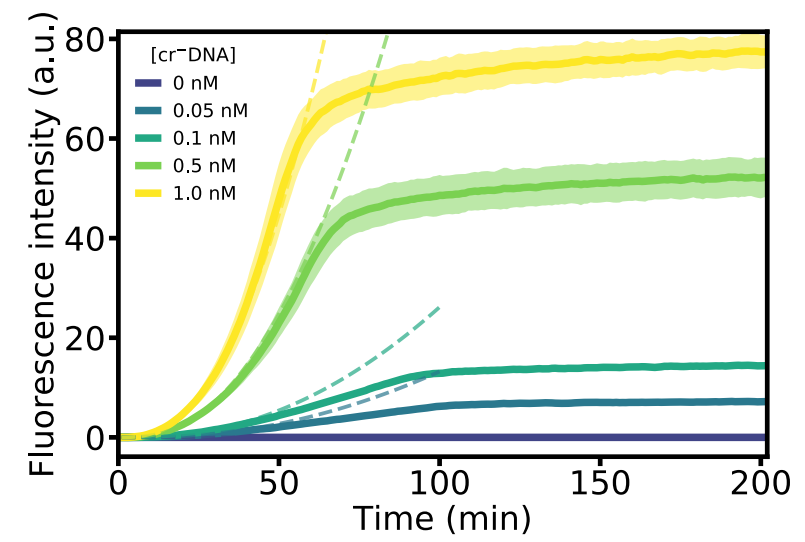

(B)

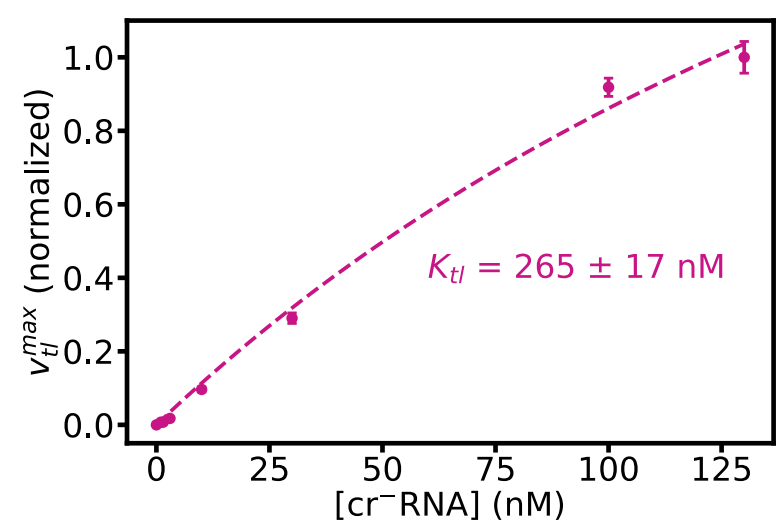

(D)

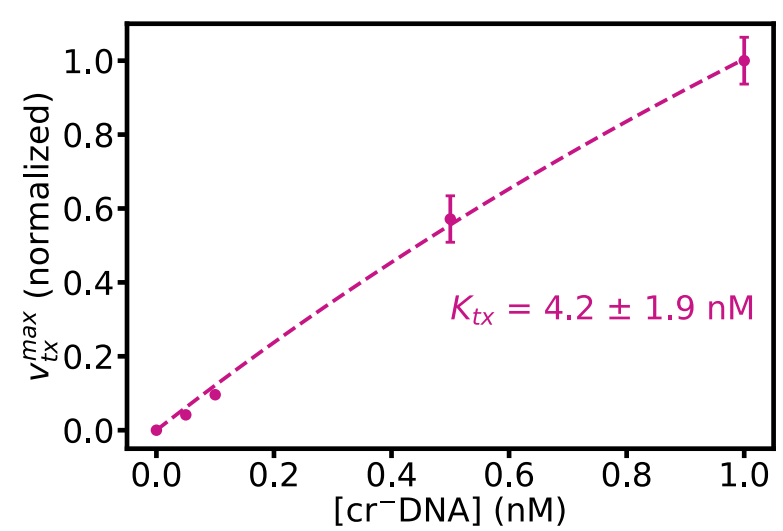

Figure 2: Characterization of the TX-TL system in the absence of riboregulation. Translation dynamics (A) and maximum fluorescence production rate (B) for increasing concentrations of an unregulated mRNA fragment coding for GFP. Expression (transcription and translation) dynamics (C) and maximum fluorescence production rate (D) for increasing concentrations of an unregulated linear DNA fragment coding for GFP. Solid lines (A,C) and disks $(\mathrm{B}, \mathrm{D})$ represent data, dotted lines are fits to the model. All experiments were performed in triplicate. Shading around the lines and error bars correspond to one standard deviation. 
and a plateau. However, the delay observed before an increase of fluorescence was now of 15 min. Finally, the quantity of DNA required to saturate the maximum rate of fluorescence growth, $v_{t x}^{\max }$, was almost two orders of magnitude lower than the quantity of RNA that saturated translation (Figure 2D).

We propose a simple analytical kinetic model that fits our data. To take into account the saturation of the production rates we assigned Michaelis-Menten kinetics to the transcription and the translation reactions. As a plausible source of the initial delay in the translation reaction, we included a first-order step of maturation of the non-fluorescent GFP protein, noted $\mathrm{P}$, into the functional fluorescent protein $P^{*}$. This is in accordance to published maturation times. ${ }^{39}$ We neglected DNA and RNA degradation and we did not take into consideration the depletion of resources because we analyzed our data between 0 and 50 min. For these reasons, our model did not reach a plateau in $P^{*}$ concentration (Figure 2A,C). These approximations are valid as long as the RNA molecules do not deteriorate and the enzymatic resources, more specifically the ribosomes, are not depleted. We thus write the following mechanism

$$
\begin{array}{rll}
\mathrm{D}_{a c t} & \stackrel{r_{\text {tz }}}{\longrightarrow} & \mathrm{D}_{a c t}+\mathrm{R}_{a c t} \\
\mathrm{R}_{a c t} & \stackrel{r_{t b}}{\longrightarrow} & \mathrm{R}_{a c t}+\mathrm{P} \\
\mathrm{P} & \stackrel{r_{m}}{\longrightarrow} & \mathrm{P}^{*}
\end{array}
$$

where $\mathrm{D}_{a c t}$ and $\mathrm{R}_{a c t}$ are, respectively, $\mathrm{cr}^{-} \mathrm{DNA}$ and $\mathrm{cr}^{-} \mathrm{RNA}$ and $r_{t x}, r_{t l}$ and $r_{m}$ are, respectively, the transcription, translation and maturation rates. With the aforementioned hypotheses, this mechanism is associated with the rate equations 


$$
\begin{aligned}
\frac{d R_{a c t}}{d t} & =r_{t x}=\frac{k_{t x} \cdot D_{a c t}}{K_{t x}+D_{a c t}} \\
\frac{d P}{d t} & =r_{t l}-r_{m}=\frac{k_{t l} \cdot R_{a c t}}{K_{t l}+R_{a c t}}-k_{m} \cdot P \\
\frac{d P^{*}}{d t} & =r_{m}=k_{m} \cdot P
\end{aligned}
$$

where $k_{x}$ and $K_{x}$ are, respectively, the rate and the Michaelis-Menten constants of reaction $x$ and species concentrations are noted in italics. Equations (4-6) have exact solutions both for initial conditions corresponding to the translation $\left(D_{a c t}(0)=0, R_{a c t}(0) \neq 0\right)$ and expression experiments $\left(D_{a c t}(0) \neq 0, R_{a c t}(0)=0\right)$ (SI Section 3). For translation we obtain (SI Section $3.1)$

$$
P^{*}(t)=\frac{R_{a c t}(0)}{K_{t l}+R_{a c t}(0)} \frac{k_{t l}}{k_{m}}\left(e^{-k_{m} t}+k_{m} t-1\right)
$$

The term $\left(e^{-k_{m} t}-1\right)$, due to protein maturation, makes the kink of the curves in Figure $2 \mathrm{~A}$ at $t=10 \mathrm{~min}$, while the linear term in time dominates for $t=20-50 \mathrm{~min}$. Note that when the ribosome is not saturated, $R_{a c t}(0) \ll K_{t l}$, and for $t \gg k_{m}^{-1}$ we can write

$$
P^{*}(t) \approx \frac{k_{t l}}{K_{t l}} \cdot R_{a c t}(0)
$$

explicitly showing that translation acts as a linear amplifier of the initial concentration of active RNA.

For expression, the exact solution is given in SI Section 3.3. Here we provide an approximated solution when $R_{a c t}(t) \ll K_{t l}$ (SI Section 3.2),

$$
P^{*}(t) \approx \frac{D_{a c t}(0)}{K_{t x}+D_{a c t}(0)} \frac{k_{t x} k_{t l}}{2 K_{t l}}\left(t^{2}-\frac{2}{k_{m}} t+\frac{2}{k_{m}^{2}}\left(1-e^{-k_{m} t}\right)\right)
$$

Again, if $D_{a c t}(0) \ll K_{t x}$ and $t \gg k_{m}^{-1}$, expression quadratically amplifies $D_{a c t}(0)$ into a fluorescence signal. 
Considering that the fluorescence intensity is proportional to $P^{*}$ we fitted (7) and (9) to the data in Figure 2. We obtained $K_{t x}=4.2 \pm 1.9 \mathrm{nM}, K_{t l}=265 \pm 17 \mathrm{nM}$ and $k_{m}=$ $0.10 \pm 0.01 \mathrm{~min}^{-1}$, in fair agreement with previous measurements reporting $K_{t x}=4-9 \mathrm{nM}$ for T7 RNAP, ${ }^{33,34,40} K_{t l}=66 \mathrm{nM}^{33}$ and $k_{m}=0.2 \mathrm{~min}^{-1} .{ }^{33,41}$ Note that although cell-extract TX-TL uses E. coli instead of T7 RNAP, the reported ${ }^{41}$ value of $K_{t x}$ is similar, $1-10 \mathrm{nM}$.

In summary, the saturation of transcription by DNA occurs at a concentration two-orders of magnitude lower than the saturation of translation by RNA. Below saturation, the TXTL system acts as a linear amplifier of the concentration of active RNA, $\mathrm{R}_{a c t}$, and as a quadratic amplifier of $D_{\text {act }}$ with a readout of intensity fluorescence. As a result we can use GFP fluorescence as a measure of the concentration of $\mathrm{R}_{\text {act }}$.

\section{Analytical model of translational riboregulation and quantitative definition of the dynamic range of a riboregulator}

When riboregulators are used in vivo the DNA sequences $\mathrm{D}_{c r}$ and $\mathrm{D}_{t a}$, respectively coding for the cis-repressed and trans-activator $\mathrm{RNA} \mathrm{R}_{c r}$ and $\mathrm{R}_{t a}$, can either be inserted in the chromosome, in the same plasmid or in two different plasmids. The performance of a riboregulator in vivo is assayed by fusing $\mathrm{D}_{c r}$ with a GFP and measuring the dynamic range, defined as

$$
\rho_{\text {ON/OFF }}=\frac{\text { GFP fluorescence in the presence of } \mathrm{D}_{t a}}{\mathrm{GFP} \text { fluorescence in the absence of } \mathrm{D}_{t a}}
$$

In vivo it is common to use a two-plasmid strategy, ${ }^{20,42}$ trying to improve $\rho_{O N / O F F}$ by inserting $\mathrm{D}_{t a}$ in a high-copy plasmid. The simplicity of in vitro TX-TL allows to provide a quantitative definition of $\rho_{O N / O F F}$ and to test the effect of $\mathrm{D}_{t a}$ concentration on $\rho_{O N / O F F}$. We start by writing the simplest model of riboregulation dynamics from DNA that is consistent with the results of the previous section. Within the TX-TL system the two DNA molecules, $\mathrm{D}_{c r}$ and $\mathrm{D}_{t a}$, are transcribed into the corresponding RNA strands, which associate into a 
coding RNA, $\mathrm{R}_{a c t}$. The production of $\mathrm{P}$ mainly comes from the translation of $\mathrm{R}_{a c t}$ but also may come from $\mathrm{R}_{c r}$, when cis-repression is not very effective. We thus write the following mechanism,

$$
\begin{array}{rll}
\mathrm{D}_{c r} \stackrel{r_{t x}^{c r}}{\rightarrow} \mathrm{D}_{c r}+\mathrm{R}_{c r} & , \quad \mathrm{D}_{t a} \stackrel{\stackrel{r_{t a}^{t a}}{\rightarrow}}{\rightarrow} \mathrm{D}_{t a}+\mathrm{R}_{t a} \\
\mathrm{R}_{c r}+\mathrm{R}_{t a} & \stackrel{K_{d}}{\rightleftharpoons} \mathrm{R}_{a c t} \\
\mathrm{R}_{c r} \stackrel{r_{t l}^{c r}}{\rightarrow} \mathrm{R}_{c r}+\mathrm{P} & , \quad \mathrm{R}_{a c t} \stackrel{r_{t l}^{a c t}}{\rightarrow} \mathrm{R}_{a c t}+\mathrm{P} \\
\mathrm{P} & \stackrel{r_{m}}{\rightarrow} \mathrm{P}^{*}
\end{array}
$$

We model reactions (11-14) with the following set of ODEs, that takes into account the competition for transcriptional resources,

$$
\begin{aligned}
\frac{d R_{c r}}{d t} & =r_{t x}^{c r}=\frac{k_{t x}^{c r} \cdot D_{c r}}{K_{t x}+D_{c r}+D_{t a}} \\
\frac{d R_{t a}}{d t} & =r_{t x}^{t a}=\frac{k_{t x}^{t a} \cdot D_{t a}}{K_{t x}+D_{c r}+D_{t a}} \\
R_{a c t} & =\frac{R_{c r} R_{t a}}{K_{d}} \\
\frac{d P}{d t} & =r_{t l}^{a c t}+r_{t l}^{c r} \approx \frac{k_{t l}^{a c t}}{K_{t l}} R_{a c t}+\frac{k_{t l}^{c r}}{K_{t l}} R_{c r} \\
\frac{d P^{*}}{d t} & =r_{m}=k_{m} \cdot P
\end{aligned}
$$

where we have assumed that $\mathrm{D}_{c r}$ and $\mathrm{D}_{t a}$ may have different transcription rate constants $k_{t x}^{i}$. We have also assumed, as previously, that transcription follows Michaelis-Menten dynamics, that translation can be considered a non-saturated Michaelis-Menten $\left(R_{c r}, R_{a c t} \ll k_{t l}^{a c t}\right)$ and further that the hybridization reaction (12) is fast compared with the others and thus can be considered at equilibrium. We have seen in the previous section that maturation reaction (14) introduces an additional term $\left(e^{-k_{m} t}-1\right)$ that vanishes when $t \gg k_{m}^{-1}=10$ min. To 
facilitate subsequent calculations we will suppose $t \gg k_{m}^{-1}$ and thus $P=P^{*}$.

We define $\alpha=\frac{D_{t a}}{D_{c r}}$ and integrate (15-18) to obtain (SI Section 4)

$$
P^{*}(\alpha)=\frac{k_{t l}^{a c t} \alpha}{3 K_{d} k_{t l}^{a c t}} \frac{k_{t x}^{t a} k_{t x}^{c r}}{\left(1+\alpha+\frac{K_{t x}}{D_{c r}}\right)^{2}} t^{3}+\frac{k_{t l}^{c r}}{2 k_{t l}^{a c t}} \frac{k_{t x}^{c r}}{1+\alpha+\frac{K_{t x}}{D_{c r}}} t^{2}
$$

where we have indicated explicitly that $P^{*}$ is a function of $\alpha$. We can naturally define the dynamic range of the riboregulator as

$$
\rho_{O N / O F F}^{t h}=\frac{P^{*}(\alpha)}{P^{*}(\alpha=0)}=\frac{1+\frac{K_{t x}}{D_{c r}}}{\left(1+\alpha+\frac{K_{t x}}{D_{c r}}\right)}\left[1+\frac{2}{3} \frac{k_{t x}^{t a} k_{t l}^{a c t}}{K_{d} k_{t l}^{c r}} \frac{\alpha}{\left(1+\alpha+\frac{K_{t x}}{D_{c r}}\right)} t\right]
$$

where the superscript th indicates that this is an theoretical quantity defined in the framework of model (15-18). This equation reveals three important points. Firstly, $\rho_{O N / O F F}^{t h}$ depends linearly on time and thus it is difficult to compare $\rho_{O N / O F F}$ between two experiments, in vivo or in vitro, if they have not been calculated at the same time. This linear dependence comes from the fact that protein production from the riboregulator is cubic in time, while the leak production from $D_{c r}$ alone is quadratic (20). In vivo one may expect that $\rho_{O N / O F F}^{t h}$ reaches a plateau due to degradation. However a linear increase of $\rho_{O N / O F F}$ was observed over $4 \mathrm{~h}$ for riboregulator 7 in reference 20. Secondly, $\rho_{O N / O F F}^{t h}$ is proportional to the aggregate factor $\beta=\frac{k_{t x}^{t a} k_{t l}^{a c t}}{K_{d} k_{t l}^{c r}}$. Thus, $\rho_{O N / O F F}^{t h}$ is proportional to the transcription rate constant $k_{t x}^{t a}$, and thus will differ between different RNAPs and promoters, it is also proportional to the ratio between the translation rate constant of the active and the inactive state, which is intuitive, and it is inversely proportional to the equilibrium constant of dissociation between $\mathrm{R}_{c r}$ and $\mathrm{R}_{t a}$. Finally, $\rho_{O N / O F F}^{t h}$ is strongly and non-trivially dependent on the concentration of $\mathrm{D}_{c r}$ and $\mathrm{D}_{t a}$ (through $\alpha=D_{t a} / D_{c r}$ ). This is, to the best of our knowledge, the first rigorous definition of the dynamic range of a translational riboregulator. The maximum of $\rho_{O N / O F F}^{t h}$ is obtained for (SI Section 4.2)

$$
D_{t a}=D_{c r}+K_{t x}
$$


(A)

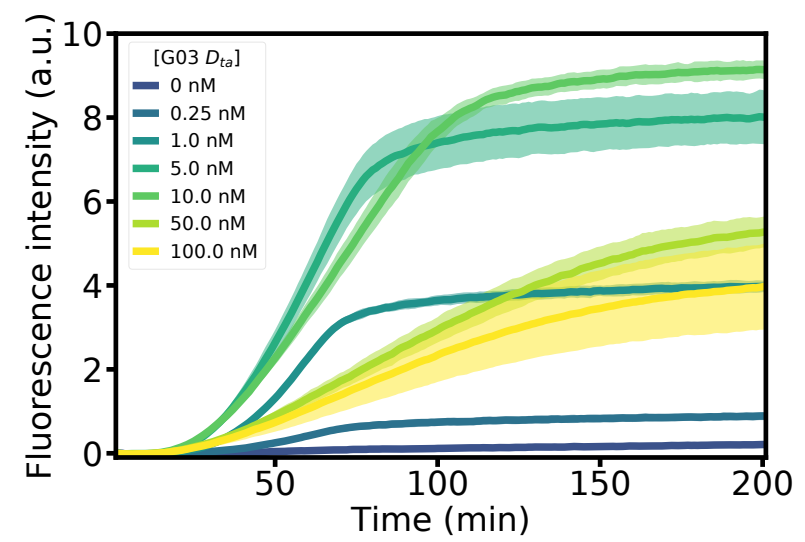

(B)

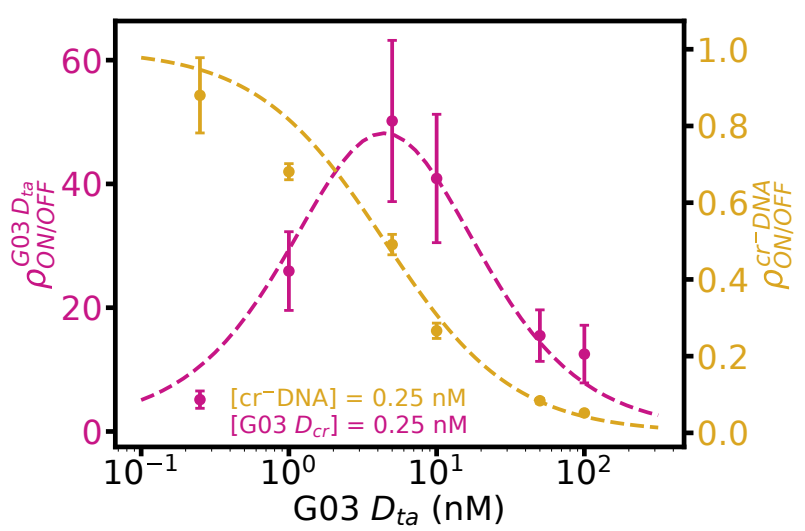

Figure 3: The dynamic range of a riboregulator strongly depends on the concentration of $D_{t a}$. (A) Fluorescence intensity vs. time for the in vitro expression of $0.25 \mathrm{nM}$ of $\mathrm{D}_{c r} \mathrm{DNA}$, coding for GFP, with increasing concentrations of $\mathrm{D}_{t a}$ DNA, for riboregulator G03. (B) Dynamic range $\rho_{O N / O F F}$ at time $75 \mathrm{~min}$ for a $\mathrm{D}_{c r}$ with, G03 (pink disks, left axis), or without, $\mathrm{cr}^{-}$ (yellow disks, right axis), cis regulatory region as a function of the concentration of $\mathrm{D}_{t a}$ from riboregulator G03. The dashed lines correspond to a fit to equation (21) with a single free parameter $\beta=\left(k_{t x}^{t a} k_{t l}^{a c t}\right) /\left(K_{d} k_{t l}^{c r}\right)$ (pink) and to SI equation (30) without fit (yellow), both using $K_{t x}=4.2 \mathrm{nM}$ as measured in Figure 2D. All experiments were performed in triplicate. Shading around the lines and error bars correspond to one standard deviation.

To test the model's prediction that $\rho_{O N / O F F}$ strongly depends on $\alpha$ and thus on $D_{t a}$, we titrated riboregulator G03 (Table S1) by keeping $D_{c r}=0.25 \mathrm{nM}$ constant, varying $D_{t a}$ in the range $0-100 \mathrm{nM}$ and recording GFP fluorescence over time (Figure 3). Increasing $D_{t a}$ in the range $0-5 \mathrm{nM}$ resulted in an increased fluorescence signal. However, for $D_{t a}>$ $5 \mathrm{nM}$ the fluorescence signal dramatically decreased until reaching $10 \%$ of the maximum production rate at $D_{t a}=100 \mathrm{nM}$. Equation (21) fits the data with a single free parameter $\beta=286$ (Figure 3B, pink line), indicating that the bell-like shape of $\rho_{O N / O F F}$ arises from the competition of $\mathrm{D}_{t a}$ and $\mathrm{D}_{c r}$ for transcriptional resources. To further test this interpretation we titrated $\mathrm{cr}^{-} \mathrm{DNA}$, which lacks the cis-regulatory region, with the $\mathrm{D}_{t a}$ of riboregulator G03. The data were quantitiatively predicted by equation (21) taking the limit $K_{d} \rightarrow \infty$ (SI equation (30)) without fitting parameters. Importantly, the addition of non-transcribing 
DNA had little effect on the expression dynamics (SI Figure S3).

The observation that an increase in non-coding DNA concentration reduces protein expression in TX-TL systems has already been reported ${ }^{25,30,43,44}$ and it has been modeled ${ }^{45}$ in the absence of riboregulation, although a quantitative comparison between the model and the data has not been reported. Our model (15-18) explicitly takes into account the competition between the two DNA substrates $\mathrm{D}_{t a}$ and $\mathrm{D}_{c r}$ and the predicted dependence of $\rho_{O N / O F F}^{t h}$ on $D_{t a}$ is in agreement with the data. Hu et al. recently proposed a kinetic model for transcriptional riboregulators ${ }^{31}$ and compared their model with in vitro TX-TL experiments. Our models are of the same type in the sense that they describe the kinetics with a set of ODEs at the level of concentrations. In contrast, the model of $\mathrm{Hu}$ et al has significantly more parameters than ours, 13 instead of 5, and takes into account the degradation of both RNA and protein. As a result, the authors cannot provide analytical results that clearly pinpoint the important parameters to design functional riboregulators, such as equation (21).

Saturation of transcriptional resources is particularly important in the context of riboregulators, where the non-coding DNA produces a regulatory RNA that has an important effect in the GRN. To the best of our knowledge, the bell-like curve in Figure 3B has not been reported before. The similar value of $K_{t x}$ for T7 and E. coli RNAP, together with previous observations of transcriptional saturation in E. coli-based TX-TL systems, suggests that this behavior is not due to a particular property of the T7 RNAP. Our model and in vitro results thus predict that inserting $\mathrm{D}_{t a}$ in a high-copy plasmid will decrease the performance of the riboregulator activator and suggests a trade-off between resource competition and the over-expression of antisense RNAs. This prediction shall be tested in a future work. 


\section{Comparison of the dynamic range of riboregulators in vivo and in} vitro

To evaluate how dynamic ranges determined in vitro compared with in vivo measurements, we investigated five riboregulators of two different types, two loop-mediated ${ }^{21}$ and three toehold-mediated $^{20}$ (Table S1). In the former, the RBS is buried inside the hairpin and the $\mathrm{R}_{t a}$ binds first to the loop on the hairpin. In the later, the start codon is protected by the hairpin and the $\mathrm{R}_{t a}$ binds to a toehold sequence on the 5' side of the hairpin (Figure 1B). We performed in vitro GFP expression experiments at $1 \mathrm{nM} \mathrm{D}_{c r}$ in the presence and in the absence of $5 \mathrm{nM}$ of the corresponding $\mathrm{D}_{t a}$. We chose $D_{c r}$ and $D_{t a}$ that verified (22) to determine the maximum dynamic range. In vivo, the dynamic range is generally defined without subtracting the autofluorescence of the cells. ${ }^{20,21}$ In the previous section, to compare with $\rho_{O N / O F F}^{t h}$, we computed the experimental $\rho_{O N / O F F}$ by dividing fluorescence signals that had been subtracted from the autofluorescence of the PURE system. In this section, to compare with in vivo measurements, we computed $\rho_{O N / O F F}^{\prime}$, where the prime indicates that autofluorescence was not subtracted.

Table 1: Comparison of the performance of five riboregulators in vivo and in vitro. Dynamic range calculated without subtracting the autofluorescence, $\rho_{O N / O F F}^{\prime}$, in vivo and in TX-TL, $\mathrm{ON}$ and OFF raw fluorescence signals, $I_{O N}$ and $I_{O F F}$, in TX-TL, and ratio of $\rho_{O N / O F F}^{\prime}$ in vivo relative to in vitro. In vivo data were extracted from ref. 20 for GXX and from ref. 21 for RAJXX. TX-TL data were measured at $D_{c r}=1$ and $D_{t a}=5 \mathrm{nM}$ at $t=75 \mathrm{~min}$. The typical value of autofluorescence was $0.07 \pm 0.01$ a.u.. Error bars correspond to one standard deviation of a triplicate experiment.

\begin{tabular}{cccccc}
\hline in vivo & TX-TL & TX-TL & TX-TL & ratio $\rho_{O N / O F F}^{\prime}$ \\
Device & $\rho_{\text {ON/OFF }}^{\prime}$ & $\rho_{\text {ON/OFF }}^{\prime}$ & $I_{O N}$ (a.u.) & $I_{O F F}$ (a.u.) & in vivo/ TX-TL \\
\hline G01 & $290 \pm 20$ & $37 \pm 10$ & $116 \pm 25$ & $3.1 \pm 0.5$ & $8 \pm 2$ \\
G03 & $260 \pm 30$ & $26 \pm 6$ & $81 \pm 10$ & $3.1 \pm 0.6$ & $10 \pm 3$ \\
G80L18 & $500 \pm 150$ & $23 \pm 3$ & $93 \pm 8$ & $4.1 \pm 0.4$ & $22 \pm 8$ \\
RAJ11 & $11 \pm 2$ & $1.9 \pm 0.3$ & $2.1 \pm 0.3$ & $1.1 \pm 0.1$ & $6 \pm 1$ \\
RAJ12 & $8 \pm 1$ & $1.2 \pm 0.1$ & $1.2 \pm 0.2$ & $1.1 \pm 0.1$ & $7 \pm 1$ \\
\hline
\end{tabular}

The agreement between the values obtained in vivo and in vitro is remarkable. Of course, the absolute values of $\rho_{O N / O F F}^{\prime}$ in vivo and in vitro are different, which is expected because 
$\rho_{O N / O F F}^{t h}$ is proportional to time and in vivo and in vitro data were obtained at different times (Table 1). In contrast, the relative order of $\rho_{O N / O F F}^{\prime}$ is similar in vivo and in vitro. Moreover the ratio between the two is constant for all riboregulators except for G80L18 that is twice more active in vivo, indicating that TX-TL experiments predict well $\rho_{O N / O F F}^{\prime}$ in vivo. The measured value of $\rho_{O N / O F F}^{\prime}$ for RAJ12 is close to unity. However, increasing the DNA concentrations to $D_{c r}=D_{t a}=50 \mathrm{nM}$, which increases protein production (20) while respecting (22), demonstrated that RAJ12 was indeed functional and we obtained $\rho_{O N / O F F}^{\prime}=7$ at these concentrations. Comparing the values of $I_{O F F}$ shows that RAJXX leaked significantly less than GXX while G80L18 leaked slightly more than G01 and G03. Finally, our experiments show that the remarkable $\rho_{O N / O F F}^{\prime}$ values of GXX devices come from their high $I_{O N}$, and thus a very active ON state. We thus conclude that in vitro TXTL provides values of $\rho_{O N / O F F}^{\prime}$ that correlate well with in vivo measurements, in agreement with previous reports comparing protein expression in vivo and in vitro. ${ }^{30,45,46}$

\section{Translation from RNA characterizes the reaction between the cis- repressed and the trans-activator RNA}

The regulatory step of translational riboregulators takes place when the two RNA fragments, $\mathrm{R}_{c r}$ and $\mathrm{R}_{t a}$, hybridize and thereby change the accessibility of the ribosome to a site needed for initiating translation (RBS or AUG). The core of the riboregulation process can thus be described with reactions (12) and (13), where the first one involves the hybridization of $\mathrm{R}_{c r}$ with $\mathrm{R}_{t a}$ to form an active RNA complex, $\mathrm{R}_{a c t}$, that can be translated, and the second being the translation of $\mathrm{R}_{\text {act }}$ into protein $\mathrm{P}$. We have seen that the thermodynamics of the first reaction play an important role in $\rho_{O N / O F F}^{\text {th }}$ through $K_{d}(21)$. However $K_{d}$ is not straightforward to determine. One possibility is to use an electrophoretic mobility shift assay in a polyacrylamide gel. Another way uses the property of a reverse transcriptase to terminate on stable RNA duplexes. ${ }^{13}$ In both cases these assays characterize the species $\mathrm{R}_{\text {act }}$ for being a duplex RNA but they are not sensitive to its translational activity. Here, instead, 
(A)

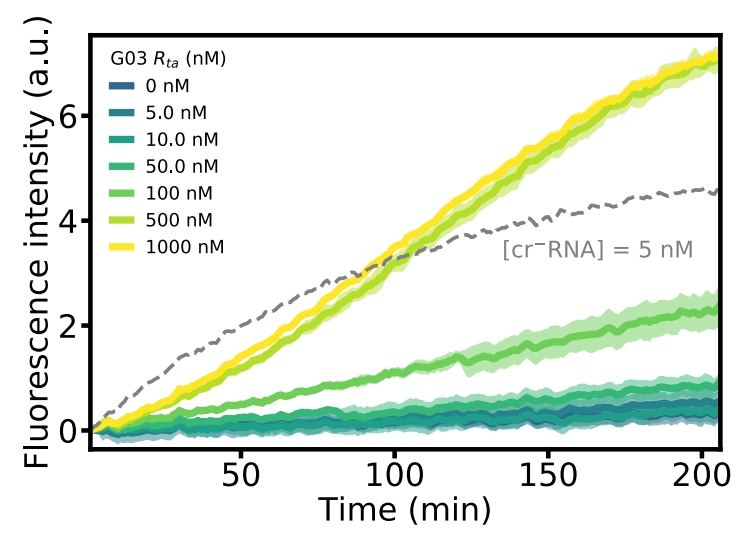

(B)

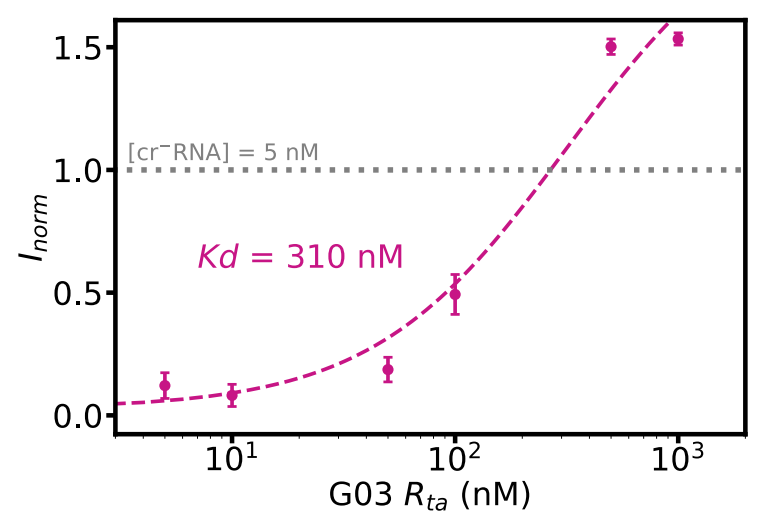

Figure 4: Titration of a riboregulator at the RNA level measures the dissociation constant of the riboregulator complex. GFP fluorescence produced over time (A) and normalized maximum fluorescence (B) for different trans-activator concentrations, $R_{t a}$ for riboregulator G03. As a control, panel A shows the fluorescence intensity produced by the translation of 5 $\mathrm{nM}$ of an unregulated $\mathrm{cr}^{-} \mathrm{RNA}$ (grey dashes). In (B) disks correspond to experimental data and the dashed line is a fit of (23) to the data. All experiments were performed in triplicate. Shading around the lines and error bars correspond to one standard deviation. 
we probed the equilibrium concentration of $\mathrm{R}_{\text {act }}$ that is active for translation. Our method is thus more meaningful to evaluate the design performances of a riboregulator.

To characterize reaction (12) we in vitro transcribed the five riboregulators described previously (Figure S1). We studied their translation dynamics by titrating $5 \mathrm{nM} \mathrm{R}_{c r}$ with increasing concentrations of its corresponding $\mathrm{R}_{t a}$ in the range $0-1000 \mathrm{nM}$ (Figure 4 and SI Figure S4). Because translation linearly amplifies $R_{\text {act }}$ (Figure 2B and (8)), measuring the GFP intensity at a given time is directly proportional to the concentration of $\mathrm{R}_{\text {act }}$ that is translationally active. We thus plotted the normalized GFP fluorescence at $200 \mathrm{~min}$ as a function of the $\log$ of $\mathrm{R}_{t a}$ concentration. For a bimolecular equilibrium such as (12) one expects these plots to be described by

$$
I_{\text {norm }} \sim \bar{R}_{a c t}=\frac{1}{2} R_{c r}^{0}\left(\frac{K_{d}+R_{c r}^{0}+R_{t a}^{0}}{R_{c r}^{0}}-\sqrt{\left(\frac{K_{d}+R_{c r}^{0}+R_{t a}^{0}}{R_{c r}^{0}}\right)^{2}-4 \frac{R_{t a}^{0}}{R_{c r}^{0}}}\right)
$$

where $\bar{R}_{a c t}$ is the equilibrium concentration of $\mathrm{R}_{a c t}$ and superscript 0 indicates initial concentrations (SI Section 6). Our experimental data followed this trend (Figures 4 and S4). We thus fitted (23) to the data and found dissociation equilibrium constants in the range $10-2000 \mathrm{nM}$ (Table 2), in agreement with $K_{d}$ values of the order of $100 \mathrm{nM}$ that have already been reported for loop-mediated activators. ${ }^{13}$ Values of $K_{d}$ obtained from different batches of PURE were within $50 \%$ (Figure S5).

In the case of G01, however, after a normal sigmoidal increase of $I_{\text {norm }}$ vs. $R_{t a}, I_{\text {norm }}$ decreased for $R_{t a}>200 \mathrm{nM}$ (Figure S4). To evaluate why in this particular case high $R_{t a}$ inhibited translation, we performed a control experiment where a well-behaved regulator, G80L18, activated with $50 \mathrm{nM}$ of its corresponding $\mathrm{R}_{t a}$, was titrated with increasing concentrations of $\mathrm{R}_{t a}$-G01 (Figure 5). We observed again that very high concentrations of $\mathrm{R}_{t a}$-G01 significantly reduced the final GFP concentration. In contrast, similarly high concentrations of $\mathrm{R}_{t a}$-RAJ11 did not have a significant effect in translation. We thus concluded that $\mathrm{R}_{t a}$-G01 poisoned the translation machinery, which could occur by nonspecific binding to other RNA 
components, including tRNAs, ribosomes or mRNA, with about $1 \mu \mathrm{M}$ affinity. However, a sequence alignment between riboregulator's sequence and tRNA and rRNA from E. coli did not show significant differences among riboregulators (Figure S6).

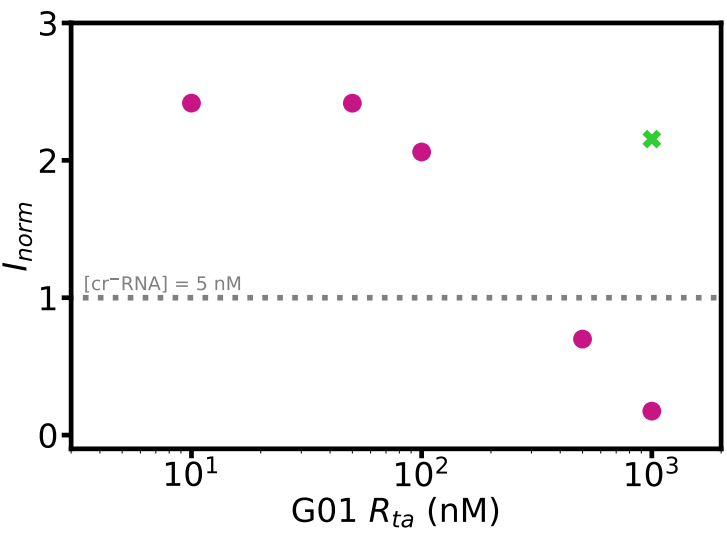

Figure 5: Titration of activated riboregulator G80L18 $\left(R_{c r}=5 \mathrm{nM} R_{t a}=50 \mathrm{nM}\right)$ with increasing concentrations of $\mathrm{R}_{t a}$ from riboregulators G01 (disks) and RAJ11 (green cross).

To assess the performance of our method for measuring $K_{d}$, we independently measured it with a standard mobility-shift assay performed with capillary gel electrophoresis. We used the same purified $\mathrm{R}_{c r}$ and $\mathrm{R}_{t a}$ that we mixed together at $37^{\circ} \mathrm{C}$ in a buffer with identical salt composition than the TX-TL system during 10 min before performing the electrophoresis assay. $\mathrm{R}_{c r}$ concentration was $8.3 \mathrm{nM}$ and the $\mathrm{R}_{t a}$ concentration was ranging from 0 to 200 nM. Figures 6 and S7 show the electropherograms for riboregulator G03, where a peak in intensity at a given time point corresponds to an RNA structure. We detected three main peaks corresponding to $\mathrm{R}_{t a}$ at $28 \mathrm{~s}$ (Figure $\mathrm{S} 7$ ) and $\mathrm{R}_{c r}$ and $\mathrm{R}_{a c t}$ complex between 37 and $40 \mathrm{~s}$ (Figure 6A). Interestingly, species $\mathrm{R}_{c r}$ and $\mathrm{R}_{a c t}$ yielded well-resolved peaks for toeholdmediated but not for loop-mediated riboregulators (Figure S8), which suggests a structural difference between the two. As a result this method only provided $K_{d}$ for some but not all of the tested riboregulators, in contrast with the TX-TL method. The values obtained were of the same order of magnitude of those obtained by TX-TL. However, mobility-shift assay yielded $K_{d}$ in a narrower range of $100-250 \mathrm{nM}$, while TX-TL was able to better discriminate $K_{d}$ for the same species and provided values in the range $15-2200 \mathrm{nM}$ (Table 2). 
(A)

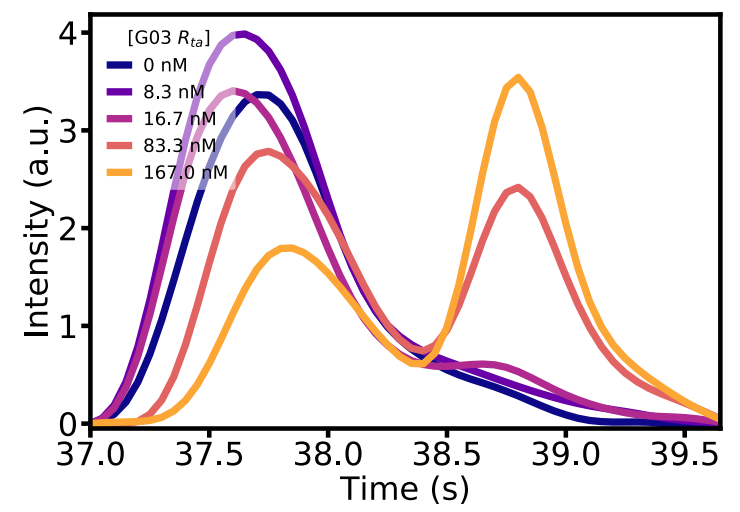

(B)

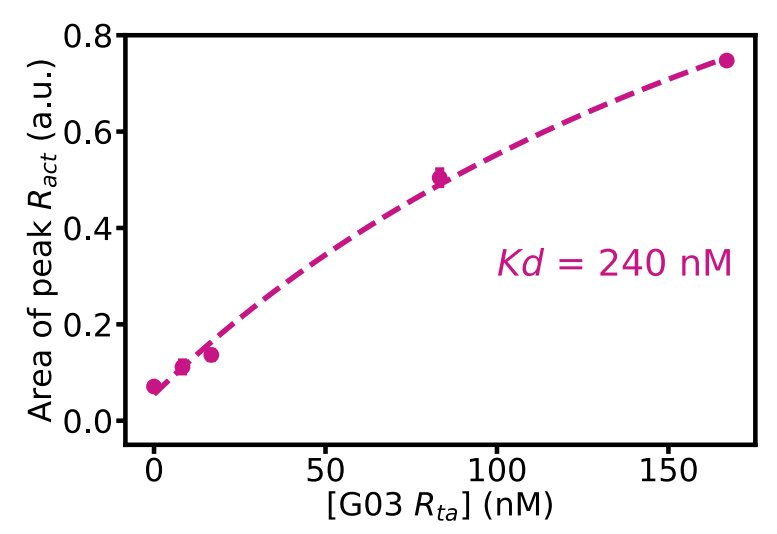

Figure 6: Titration of translational riboregulator G03 by mobility-shift capillary electrophoresis. (A) Corrected electropherograms vs. elution time and (B) peak area for different concentrations of $\mathrm{R}_{t a}$. Experiments were performed in triplicate. Error bars correspond to one standard deviation. Dashed line is a fit of (23) to the data.

Table 2: Dissociation constants $K_{d}$ at $37^{\circ} \mathrm{C}$ for the studied riboregulator devices. $K_{d}$ was measured using the cell-free translation method (txtl) and the mobility-shift method (ms). N.M. indicates that the electropherogram showed ill-defined peaks from which $K_{d}$ could not be extracted. Error bars correspond to one standard deviation of the fit. Values for G03 and RAJ11 were fitted to data in triplicate.

\begin{tabular}{ccc}
\hline Device & $K_{d}^{t x t l}(\mathrm{nM})$ & $K_{d}^{m s}(\mathrm{nM})$ \\
\hline G01 & $46 \pm 56$ & $180 \pm 20$ \\
G03 & $310 \pm 154$ & $240 \pm 110$ \\
G80L18 & $31 \pm 19$ & $110 \pm 90$ \\
RAJ11 & $15 \pm 14$ & N.M. \\
RAJ12 & $2220 \pm 950$ & N.M. \\
\hline
\end{tabular}




\section{Conclusion}

We have demonstrated that in vitro transcription-translation (TX-TL) systems are an attractive platform to quantitatively characterize translational riboregulators. To do so we have taken advantage of the ribosome as a molecular machine that not only recognizes RNA complexes that are translationally active but also measures their concentration. The simplicity of the TX-TL system allowed us to propose an analytical expression for the dynamic range of a riboregulator. In quantitative agreement with this model we have shown that increasing the DNA concentration of the trans-activating species first promotes and later inhibits expression. This result suggests that inserting trans-activating elements in high-copy plasmids in vivo could limit the efficiency of translational activators, a prediction that shall be tested in future work. Furthermore, relative dynamic ranges measured in vitro were in agreement with those reported in vivo for four out of five measured riboregulators. Finally, by titrating the cis-repressed gene with the trans-activating species at the RNA level we could determine dissociation constants, $K_{d}$, for the RNA hybridization reaction in a very simple manner. In particular, we could obtain $K_{d}$ 's for riboregulators that could not be resolved by mobility-shift assays. Our method thus provides a simple and rapid way for the quantitative characterization of riboregulators.

Combined with other biomolecular techniques such as molecular beacons ${ }^{34}$ and automatedbased designs ${ }^{47}$ cell-free transcription-translation systems are becoming essential for a wide brand of applications. They allow to verify theoretical predictions on both RNA structures and behaviour of large scale regulatory networks. Their versality is a real asset for conceiving new synthetic biological features ${ }^{48}$ and creating innovative biomolecular tools. ${ }^{49}$ The use of an in vitro step in the design and elaboration of complex synthetic regulatory networks will maximise the chance of expected in-vivo performances. 


\section{Methods}

\section{DNA and RNA preparations}

DNA templates were prepared by PCR amplification of plasmids encoding for the RNA translational regulators, followed by affinity column purification using Monarch PCR Purification Kit (New England BioLabs) or PureLink PCR Purification Kit (Thermo Fisher Scientific). Primers used for PCR amplification contained a T7 promoter or a T7 terminator (Biomers). RNA templates were prepared by in vitro transcription followed by purification using MEGAclear Transcription Clean-Up Kit (Ambion). The DNA and RNA integrity was determined by a $1.5 \%$ agarose gel (Figure S1) and the concentrations were determined by absorbance at $260 \mathrm{~nm}$ using a NanoDrop 2000 UV-Vis spectrophotometer. The sequences of the riboregulator domains (Table S1), of the PCR primers (Table S2) and of the plasmids are compiled in the SI.

\section{Preparation of the PURE TX-TL system}

The PURE TX-TL system was prepared according to reference 50 to reach the following composition: 1 units/ $\mu \mathrm{L}$ of RNase inhibitor Murine (New England Biolabs), 50 mM Hepes$\mathrm{KOH}$ pH 7.6, $13 \mathrm{mM}$ magnesium acetate, $100 \mathrm{mM}$ potassium glutamate, $2 \mathrm{mM}$ spermidine, $1 \mathrm{mM}$ dithiothreitol (DTT), $2 \mathrm{mM}$ of each ATP and GTP, $1 \mathrm{mM}$ of each CTP and UTP, $20 \mathrm{mM}$ creatine phosphate, $0.3 \mathrm{mM} 20$ amino acids, 56 A260/ml tRNA mix (Roche), 10 $\mu \mathrm{g} / \mathrm{mL}$ 10-formyl-5, 6, 7, 8-tetrahydrofolic acid, $0.1 \mathrm{mM}$ each of amino acids, and factor mix. The factor mix contained $1.2 \mu \mathrm{M}$ ribosome, $10 \mu \mathrm{g} / \mathrm{ml}$ IF $1,40 \mu \mathrm{g} / \mathrm{ml}$ IF2, $10 \mu \mathrm{g} / \mathrm{ml}$ IF3, $50 \mu \mathrm{g} / \mathrm{ml}$ EF-G, $100 \mu \mathrm{g} / \mathrm{ml}$ EF-Tu, $50 \mu \mathrm{g} / \mathrm{ml}$ EF-Ts, $10 \mu \mathrm{g} / \mathrm{ml}$ RF1, $10 \mu \mathrm{g} / \mathrm{ml}$ RF2, $10 \mu \mathrm{g} / \mathrm{ml} \mathrm{RF} 3,10 \mu \mathrm{g} / \mathrm{ml} \mathrm{RRF}, 600-6000 \mathrm{U} / \mathrm{ml}$ of each ARS and MTF $4.0 \mu \mathrm{g} / \mathrm{ml}$ creatine kinase (Roche), $3.0 \mu \mathrm{g} / \mathrm{ml}$ myokinase (Sigma), $1.1 \mu \mathrm{g} / \mathrm{ml}$ nucleoside-diphosphate kinase, 1.0 $\mathrm{U} / \mathrm{ml}$ pyrophosphatase (Sigma), and $10 \mu \mathrm{g} / \mathrm{ml}$ of T7 RNAP. 


\section{Fluorescence measurements in real-time PCR machine}

Rotor-GeneQ real-time PCR (Qiagen) was used to record fluorescence from GFP expression (excitation $470 \pm 10 \mathrm{~nm}$, emission $510 \pm 5 \mathrm{~nm}$ ) in an 8 or $15 \mu \mathrm{L}$ volume. The temperature was set to $37^{\circ} \mathrm{C}$ and fluorescence recorded every minute for at least $3 \mathrm{~h}$. In some experiments (Figure 5) we used PURExpress in vitro protein synthesis kit (NEB).

\section{Data processing}

Data were processed using in-house Python routines. For each condition of template — DNA or RNA - concentration, fluorescence intensity plots were shifted to the origin by removing the mean value of the three first minutes and by subtracting the fluorescence due to the PURE TX-TL system without any template. $I_{\text {norm }}$ was computed by dividing this corrected fluorescence by the final intensity of the $\mathrm{cr}^{-}$RNA control. Corrected data were filtered using a Savitzky-Golay filter (window length: 21, polynomial order: 3) to remove residual noise before being derived to compute $v^{\max }$.

\section{Electrophoretic mobility shift assays}

Electrophoretic mobility shift assays were performed with a 2100 Bioanalyzer System (Agilent Technologies) and an RNA Nano chip Kit. Samples were prepared by mixing RNA strands in 50 mM Hepes-KOH pH 7.6, 13 mM magnesium acetate, $100 \mathrm{mM}$ potassium glutamate, $2 \mathrm{mM}$ spermidine, $1 \mathrm{mM}$ DTT and nuclase free water. They were incubated at $37^{\circ} \mathrm{C}$ for 10 min before being loaded into the electrophoresis chip. Electropherograms were manually aligned along the time axis. Affine curves corresponding to the backgrounds of zones of interest were subtracted. Areas under peaks were determined by numerical integration and were normalized using an RNA marker provided in Agilent's kit. 


\section{Acknowledgement}

The authors thank H. Isambert for helpful discussions, A. Green for providing the expression plasmids coding for the toehold-mediated riboegulators and J.-C. Galas for comments on the manuscript. This research was supported by the European commission FET-Open program under award Ribonets (323987).

\section{Supporting Information Available}

Sequences for the RNA regulators, the PCR primers and the plasmids used, additional figures S1-S8, tables S1-S2, the detailed solution of translation and expression kinetics in the absence and in the presence of regulation, derivation of the equation used for $\mathrm{Kd}$ determination, supplementary data on RNA titration and details on the sequence alignment.

\section{References}

1. Gardner, T. S., Cantor, C. R., and Collins, J. J. (2000) Construction of a genetic toggle switch in Escherichia coli. Nature 403, 339-342, 10.1038/35002131.

2. Elowitz, M. B., and Leibler, S. (2000) A synthetic oscillatory network of transcriptional regulators. Nature 403, 335-338.

3. Cameron, D. E., Bashor, C. J., and Collins, J. J. (2014) A brief history of synthetic biology. Nat Rev Micro 12, 381-390.

4. Purnick, P. E. M., and Weiss, R. (2009) The second wave of synthetic biology: from modules to systems. Nat Rev Mol Cell Biol 10, 410-422, 10.1038/nrm2698.

5. Niederholtmeyer, H., Sun, Z. Z., Hori, Y., Yeung, E., Verpoorte, A., Murray, R. M., and Maerkl, S. J. (2015) Rapid cell-free forward engineering of novel genetic ring oscillators. Elife 4 . 
6. Moon, T. S., Lou, C., Tamsir, A., Stanton, B. C., and Voigt, C. A. (2012) Genetic programs constructed from layered logic gates in single cells. Nature 491, 249.

7. Das, R., and Baker, D. (2008) Macromolecular Modeling with Rosetta. Annual Review of Biochemistry 77, 363-382.

8. Schaerli, Y., Munteanu, A., Gili, M., Cotterell, J., Sharpe, J., and Isalan, M. (2014) A unified design space of synthetic stripe-forming networks. Nat Commun 5, $10.1038 /$ ncomms5905.

9. Zuker, M. (2003) Mfold web server for nucleic acid folding and hybridization prediction. Nucleic Acids Res. 31, 3406-3415, 10.1093/nar/gkg595.

10. Lorenz, R., Bernhart, S. H., Höner zu Siederdissen, C., Tafer, H., Flamm, C., Stadler, P. F., and Hofacker, I. L. (2011) ViennaRNA Package 2.0. Algorithms for Molecular Biology 6, 1-14.

11. Zadeh, J. N., Steenberg, C. D., Bois, J. S., Wolfe, B. R., Pierce, M. B., Khan, A. R., Dirks, R. M., and Pierce, N. A. (2011) NUPACK: Analysis and design of nucleic acid systems. Journal of Computational Chemistry 32, 170-173.

12. Chappell, J., Watters, K. E., Takahashi, M. K., and Lucks, J. B. (2015) A renaissance in RNA synthetic biology: new mechanisms, applications and tools for the future. Current Opinion in Chemical Biology 28, 47-56.

13. Isaacs, F. J., Dwyer, D. J., Ding, C., Pervouchine, D. D., Cantor, C. R., and Collins, J. J. (2004) Engineered riboregulators enable post-transcriptional control of gene expression. Nat Biotech 22, 841-847, 10.1038/nbt986.

14. Win, M. N., and Smolke, C. D. (2007) A modular and extensible RNA-based generegulatory platform for engineering cellular function. Proc. Natl. Acad. Sci. U. S. A. 104, 14283-14288. 
15. Salis, H. M., Mirsky, E. A., and Voigt, C. A. (2009) Automated design of synthetic ribosome binding sites to control protein expression. Nat. Biotechnol. 27, 946-950.

16. Qi, L., Larson, M. H., Gilbert, L. A., Doudna, J. A., Weissman, J. S., Arkin, A. P., and Lim, W. A. (2013) Repurposing CRISPR as an RNA-Guided Platform for SequenceSpecific Control of Gene Expression. Cell 152, 1173-1183.

17. Chang, A. L., Wolf, J. J., and Smolke, C. D. (2012) Synthetic RNA switches as a tool for temporal and spatial control over gene expression. Current opinion in biotechnology 23, 679-688.

18. Chappell, J., Takahashi, M. K., and Lucks, J. B. (2015) Creating small transcription activating RNAs. Nat. Chem. Biol. 11, 214-20.

19. Rinaudo, K., Bleris, L., Maddamsetti, R., Subramanian, S., Weiss, R., and Benenson, Y. (2007) A universal RNAi-based logic evaluator that operates in mammalian cells. Nat. Biotechnol. 25, 795-801.

20. Green, A. A., Silver, P. A., Collins, J. J., and Yin, P. (2014) Toehold switches: de-novodesigned regulators of gene expression. Cell 159, 925-39.

21. Rodrigo, G., Landrain, T. E., and Jaramillo, A. (2012) De novo automated design of small RNA circuits for engineering synthetic riboregulation in living cells. Proceedings of the National Academy of Sciences 109, 15271-15276.

22. Greenleaf, W. J., Frieda, K. L., Foster, D. A. N., Woodside, M. T., and Block, S. M. (2008) Direct Observation of Hierarchical Folding in Single Riboswitch Aptamers. Science (80-. ). 319, 630-633.

23. Ge, P., and Zhang, S. (2015) Computational analysis of RNA structures with chemical probing data. Methods 79, 60-66. 
24. Watters, K. E., Abbott, T. R., and Lucks, J. B. (2016) Simultaneous characterization of cellular RNA structure and function with in-cell SHAPE-Seq. Nucleic Acids Research 44, e12-e12, 10.1093/nar/gkv879.

25. Noireaux, V., Bar-Ziv, R., and Libchaber, A. (2003) Principles of cell-free genetic circuit assembly. Proc. Natl. Acad. Sci. U. S. A. 100, 12672-7.

26. Garamella, J., Marshall, R., Rustad, M., and Noireaux, V. (2016) The All E. coli TX-TL Toolbox 2.0: A Platform for Cell-Free Synthetic Biology. ACS Synth. Biol. 5, 344-55.

27. Sun, Z. Z., Yeung, E., Hayes, C. A., Noireaux, V., and Murray, R. M. (2014) Linear DNA for Rapid Prototyping of Synthetic Biological Circuits in an Escherichia coli Based TX-TL Cell-Free System. ACS Synth. Biol. 3, 387-397.

28. Shimizu, Y., Inoue, A., Tomari, Y., Suzuki, T., Yokogawa, T., Nishikawa, K., and Ueda, T. (2001) Cell-free translation reconstituted with purified components. Nat Biotech 19, $751-755$.

29. Shimizu, Y., Kanamori, T., and Ueda, T. (2005) Protein synthesis by pure translation systems. Methods 36, 299-304.

30. Takahashi, M. K., Chappell, J., Hayes, C. A., Sun, Z. Z., Kim, J., Singhal, V., Spring, K. J., Al-Khabouri, S., Fall, C. P., Noireaux, V., Murray, R. M., and Lucks, J. B. (2015) Rapidly Characterizing the Fast Dynamics of RNA Genetic Circuitry with CellFree Transcription-Translation (TX-TL) Systems. ACS Synthetic Biology 4, 503-515.

31. Hu, C. Y., Varner, J. D., and Lucks, J. B. (2015) Generating Effective Models and Parameters for RNA Genetic Circuits. ACS Synth. Biol. 4, 914-926.

32. Whittaker, J. W. (2013) Cell-free protein synthesis: The state of the art. Biotechnol. Lett. 35, 143-152. 
33. Stogbauer, T., Windhager, L., Zimmer, R., and Radler, J. O. (2012) Experiment and mathematical modeling of gene expression dynamics in a cell-free system. Integrative Biology 4, 494-501.

34. Niederholtmeyer, H., Xu, L., and Maerkl, S. J. (2013) Real-Time mRNA Measurement during an in Vitro Transcription and Translation Reaction Using Binary Probes. ACS Synthetic Biology 2, 411-417.

35. Niederholtmeyer, H., Stepanova, V., and Maerkl, S. J. (2013) Implementation of cell-free biological networks at steady state. Proceedings of the National Academy of Sciences

36. Gruber, A. R., Lorenz, R., Bernhart, S. H., Neuböck, R., and Hofacker, I. L. (2008) The Vienna RNA websuite. Nucleic Acids Res. 36, 70-74.

37. Herschlag, D. (1995) RNA Chaperones and the RNA Folding Problem. Journal of Biological Chemistry 270, 20871-20874.

38. Schroeder, R., Barta, A., and Semrad, K. (2004) Strategies for RNA folding and assembly. Nat Rev Mol Cell Biol 5, 908-919.

39. Bionumbers, http://bionumbers.hms.harvard.edu//bionumber.aspx?id=102972\&ver $=8$.

40. Maslak, M., and Martin, C. T. (1993) Kinetic analysis of T7 RNA polymerase transcription initiation from promoters containing single-stranded regions. Biochemistry 32, 4281-4285, PMID: 8476857.

41. Karzbrun, E., Shin, J., Bar-Ziv, R. H., and Noireaux, V. (2011) Coarse-grained dynamics of protein synthesis in a cell-free system. Phys. Rev. Lett. 106, 1-4.

42. Urban, J. H., and Vogel, J. Methods in Molecular Biology; Humana Press, 2009; pp $301-319$.

43. Shin, J., and Noireaux, V. (2010) Efficient cell-free expression with the endogenous E. Coli RNA polymerase and sigma factor 70. Journal of Biological Engineering 4, 8. 
44. Karig, D. K., Iyer, S., Simpson, M. L., and Doktycz, M. J. (2012) Expression optimization and synthetic gene networks in cell-free systems. Nucleic Acids Research 40, 3763-3774, 10.1093/nar/gkr1191.

45. Siegal-Gaskins, D., Tuza, Z. A., Kim, J., Noireaux, V., and Murray, R. M. (2014) Gene Circuit Performance Characterization and Resource Usage in a Cell-Free "Breadboard". ACS Synthetic Biology 3, 416-425.

46. Chappell, J., Jensen, K., and Freemont, P. S. (2013) Validation of an entirely in vitro approach for rapid prototyping of DNA regulatory elements for synthetic biology. Nucleic Acids Res. 41, 3471-3481.

47. Espah Borujeni, A., Mishler, D. M., Wang, J., Huso, W., and Salis, H. M. (2016) Automated physics-based design of synthetic riboswitches from diverse RNA aptamers. Nucleic Acids Res. 44, 1-13.

48. Iwane, Y., Hitomi, A., Murakami, H., Katoh, T., Goto, Y., and Suga, H. (2016) Expanding the amino acid repertoire of ribosomal polypeptide synthesis via the artificial division of codon boxes. Nat. Chem.

49. Pardee, K., Green, A. A., Ferrante, T., Cameron, D. E., DaleyKeyser, A., Yin, P., and Collins, J. J. (2014) Paper-Based Synthetic Gene Networks. Cell 159, 940-954.

50. Shimizu, Y., and Ueda, T. In Cell-Free Protein Production: Methods and Protocols; Endo, Y., Takai, K., and Ueda, T., Eds.; Humana Press: Totowa, NJ, 2010; pp 11-21. 


\section{Graphical TOC entry}

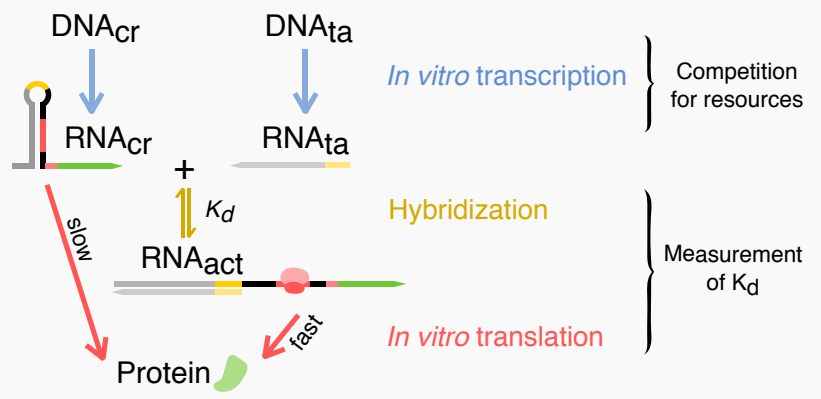

